\title{
Codex, memex, genex: The pursuit of transformational technologies
}

\author{
Ben Shneiderman \\ Professor, Department of Computer Science \\ Head, Human-Computer Interaction Laboratory \\ University of Maryland \\ College Park, MD 20742 \\ ben@cs.umd.edu
}

For: International Journal of Human-Computer Interaction

Draft December 22, 1997

\begin{abstract}
Handwritten codexes or printed books transformed society by allowing users to preserve and transmit information. Today, leather-bound volumes and illuminated manuscripts are giving way to animated image maps and hot links. Vannevar Bush's memex has inspired the World Wide Web, which provides users with vast information resources and convenient communications. In looking to the future, we might again transform society by building genexes -- generators of excellence. Such inspirational environments would empower personal and collaborative creativity by enabling users to:

- collect information from an existing domain of knowledge,

- create innovations using advanced tools,

- consult with peers or mentors in the field, and then

- disseminate the results widely.

This paper describes how a framework for an integrated set of software tools might support this four-phase model of creativity in science, medicine, the arts, and beyond. Current initiatives are positive and encouraging, but they do not work in an integrated fashion, often miss vital components, and are frequently poorly designed. A well-conceived and clearlystated framework could guide design efforts, coordinate planning, and speed development.
\end{abstract}

\section{Keywords}

genex, memex, World-Wide Web, advanced graphical user interfaces, computer supported cooperative work, information visualization

\section{Introduction}

Computer professionals have much to be proud of in the past fifty years. We have developed a scientific discipline, a remarkable technology, and a thriving industry. Computing, information, and communications technology have transformed aviation, automobiles, medicine, publishing, banking, and many more areas. These transformations will continue but as we look forward to the next fifty years, we may consider a more planful process in shaping the applications of our maturing technology and in dealing with many serious problems facing our civilization.

This essay proposes a framework to support developers of integrated families of tools that support creative problem solving. This ambitious goal may be realizable if we have an effective model of how creative people work and what tools would support their efforts. It may also help to have an historical perspective to perceive evolving patterns.

Early transformational technologies include paper and printing, which are referred to by the term "codex" in the title of this essay. Durable books and movable type reshaped science, religion, politics, and daily life in many ways. Ensuing information technology innovations included photography, telephony, radio, and television. Then, in July 1945 President Roosevelt's science adviser, Vannevar Bush (Bush 1945) described memex, for "memory extender." In his essay on grand projects that scientists might work on as World War II was coming to an end, he proposed a personal desktop system, based on microfilm technology, that would provide access to historical sources for domains such as patents, legal citations, or scientific papers. The vision of memex as a personal workstation was a powerful force in shaping the development of personal computers, our work on hypertext (Shneiderman and Kearsley, 1989), and the emergence of the World-Wide Web (Berners-Lee, 1993).

Later visions from J. C. R. Licklider helped launch digital libraries, which he saw as a centralized resource that could be accessed electronically (Licklider, 1965). Licklider was profoundly influenced by Bush's vision and dedicated his book to Bush. Douglas Engelbart's powerful notions of intelligence augmentation by advanced user interfaces and Computer Supported Cooperative Work were key inspirations for the personal computer and groupware (Engelbart, 1968). The theme of collaborative tools to support scientific research stimulated William Wulf to coin the term "collaboratories" in his 1989 white paper (NRC, 1993). 
Personal computing and network access have already become widespread in some countries, and will spread further as prices decline, user interfaces become more well designed, and services become more relevant. Universal service is an important goal to pursue, so that the benefits of computing and communications are available to all who seek them. There is a growing risk that advancing technology will widen the gap between rich and poor, and produce further disadvantages for poorly educated citizens. Progress is needed in low-cost manufacturing techniques, convenient public services, improved training, and effective support.

But even as these improvements are being made, it may be useful to think further ahead to grander goals that may constructively transform society. We should move beyond the modest goal of getting a computer to perform as

well as the best human being, and pursue tools that enable humans to perform a thousand times better than the most capable human (Shneiderman, 1997). Printing presses, photography, and electronic mail satisfy this test, but foreseeing the next innovation is difficult.

The first steps towards building new tools may already be appearing in advanced software packages and on the World Wide Web. Creative designers are introducing environments that go well beyond the vision supplied by Bush, Licklider, Engelbart, and Wulf. They prefigure tools that are more than a digital library or intelligence amplifier; they support creativity. I might call such a tool memex 2.0, to suggest an upgrade of Bush's memex, but I prefer genex, to indicate an orientation towards generating excellence. (Footnote: The closeness of genex to genetics and to generation- $X$ is coincidental.) This may be an optimistic expectation, but it conveys my belief that well-designed technologies can promote excellence. Users who experience empowering designs that are comprehensible, predictable, and controllable, may be inspired to pursue quality in their work products.

A genex would be an integrated family of direct manipulation tools that supports users in creating innovations in art, science, engineering, etc. A genex would help users initiate hopes, fabricate plans, and implement dreams in a highly social framework. It would facilitate dialog with peers and mentors, and then dissemination to potential beneficiaries. The current initiatives are positive and encouraging, but they do not work in an integrated fashion, often miss vital components, and are frequently poorly designed. A wellconceived and clearly-stated framework could guide design efforts, coordinate planning, and speed development.

Marshall McLuhan pointed out that new media are constrained by a rear-view mirror approach to their content, that is, new media depend on the old media until the unique features of the new media are appreciated. For example, the contents of early movies were novels and plays, and the contents of early television was (and often still is) movies.
Naturally the early contents of computing and the most popular user interfaces are for word processing paper documents, maintaining mailing lists, and dealing with spreadsheets. I believe that the intrinsic message of the medium of computers and user interfaces is the capacity to create. But using new technology (user interfaces) to create old media (paper documents) is still the rear-view mirror approach. The future of this media is likely to be tied to the capacity of users to create new user interfaces. Some users are doing this in modest ways by weaving web pages or composing Visual Basic programs, but there is still a large opportunity to facilitate more creative interactive experiences. Clever designers could forge more powerful tools that would enable users to invent useful visual languages, startling musical instruments, or fresh democratic processes. Future genexes could enable users to devise simulations, construct drug discovery tools, or assemble novel groupware. The design of tools for toolsmiths

(Brooks, 1996) could also be supported by a genex, but for now, let's avoid this recursive jump.

This essay is meant to guide genex designers by offering a unified conception, a clear framework, and several examples. Appropriate genex designs would enable problem solvers to locate and build on previous work easily, explore numerous alternatives rapidly, consult conveniently, and propagate solutions widely. Section 2 describes the intellectual foundation for genex technologies, Section 3 lays out the four phases of creative work, and Section 4 offers exemplary scenarios of how genexes might inspire work in several domains. Since any powerful tool, such as a genex, can be used for destructive purposes, the cautions are discussed in Section 5. Other concerns and barriers are discussed along the way.

\section{Foundational beliefs}

Linking computer science to societal transformation is difficult, but for me the attraction of such a bridge is strong. I believe that the foundations can be built from a set of beliefs about how computing contributes to creative endeavors with high societal benefits.

I start with the analysis of creativity by the psychologist Mihalyi Csikszentmihalyi who studied 91 world-famous individuals (Nobel and Pulitzer Prize winners, corporate leaders, established artists, etc.), mostly over the age of sixty (1993). He defines creative work in the context of knowledge domains created by a field of individual persons. A

domain, such as mathematics, biology, or history, consists of shared symbolic knowledge, rules and procedures. A field includes the gatekeepers of knowledge for a domain, such as curators, teachers, and scientific journal reviewers. Csikszentmihalyi writes:

Creativity occurs when a person, using the symbols of a given domain ...has a new idea or sees a new pattern, and when this novelty is selected by the appropriate field for 
inclusion in the relevant domain. The next generation will encounter that novelty as part of the domain they are exposed to, and if they are creative, they in turn will change it further.

The continuing process of learning a domain, conducting creative work within the domain (or bridging two domains and occasionally creating a new domain), receiving validation from members of the field, and contributing

to the domain is in harmony with my foundational beliefs about creative endeavors:

\section{Foundational Belief 1: \\ New knowledge is built on previous Knowledge.}

Creative individuals build new knowledge based on their understanding of existing domains. They combine their knowledge in novel ways or invent new knowledge that is useful to some field. Innovators collect relevant previous knowledge and understand it sufficiently well to build on it. Hand-written codexes (or codices) and then books have been the major source for previous knowledge, but digital storage, electronic dissemination, and display screens are rapidly becoming an appealing alternative. Resources include patents, legal statutes, photos, symphonies, and architectural drawings. In any medium, locating the relevant knowledge can be difficult, costly, and time consuming, but computing technology (a memex) can be helpful.

\section{Foundational Belief 2:}

\section{Powerful tools can support creativity.}

Innovation can be facilitated by powerful tools that supply templates and support exploratory processes such as brainstorming (offering links to related concepts), state-space exploration (trying out all permutations), idea combining (systematic pairings), rapid prototyping, and simulation modeling. If aircraft wing designers have design tools, they can try out a range of alternate wing profiles at low and high speed to maximize lift. If physicians have clinical databases, they can study treatment plans with low or high dosages of multiple medications and contrast them with surgical

outcomes for cases matching their patient's profile. If artists have drawing tools, they can explore color, texture, and repetition, to create the mood they want. Organizing creative work to apply computing tools is still an intellectual challenge, but computers may help in this early phase as well.

\section{Foundational Belief 3:}

Refinement is a social process.

New ideas are conceived of by individuals, and then they are refined through reviews from knowledgeable peers and mentors. The consultation and participatory design processes yields confirmations, suggestions, and refutationsthat lead to vital refinements. Scientific papers are refereed by journal editors and architectural plans are reviewed by clients, zoning boards, and city commissions. Consultation can be difficult because appropriate and willing peers or mentors may be hard to find, but also rewarding because the thrill of innovation is richer when respected colleagues respond favorably.

\section{Foundational Belief 4:}

\section{Creative work is not complete until it is disseminated.}

Bringing refined knowledge to scholars, practitioners, and students is central to the cumulative nature of science, art, and progress. Reaching large appropriate audiences to create discussions is currently difficult. Scientific conferences, political speeches, or symphony performances can spread innovations and spark lively debates, but only among the limited number of attendees. The current mass media such as television, books, and magazines are one-directional, and are produced by a centralized process. This can be positive, since respected editors can filter material to ensure consistency and high quality, but more widely accessible narrowcasting to specific audiences could enable livelier

decentralized discussions. Democratic processes for presenting opposing views, caucusing within factions, and finding satisfactory compromises are productive for legislative, commercial, and scholarly pursuits.

These foundational beliefs form a natural base on which to guide designers in building an integrated family of tools that support creative work. However, each belief has a dark side whose dangers must be considered. Immersion in previous work may bias creativity and limit imagination if users cannot break free from tradition. Use of powerful creativityenhancing tools may confine exploration to outcomes already envisioned. Consultation can lead to discouraging criticism by vested interests or theft of an idea by powerful and wellpositioned organizations. Dissemination can be financially demanding and anxiety producing, as solitary problem solving gives was to highly visible promotional presentations. Dissemination may also be restricted because many organizations wish to limit publication of innovations so as to protect their competitive advantages.

The next section describes the four phases of creativity and the tools that might be included in a genex (Table 1). Inevitably there will be many genexes; oriented towards different domains and tailored to the needs of different user communities, such as eager students acquiring a domain, methodical workers in established sub-domains, or visionary explorers of new domains. 


\begin{tabular}{|l|l|l|}
\hline Foundational Beliefs & Creative Phases & Genex Tools \\
\hline $\begin{array}{l}\text { 1) } \begin{array}{l}\text { New knowledge is built on } \\
\text { previous knowledge }\end{array} \\
\text { 2) } \begin{array}{l}\text { Powerful tools can support } \\
\text { Creativity }\end{array}\end{array}$ & $\begin{array}{l}\text { Collect information from an } \\
\text { existing domain of knowledge } \\
\text { Create innovations using }\end{array}$ & $\begin{array}{l}\text { Digital libraries } \\
\text { Search services } \\
\text { Information visualization } \\
\text { Multimedia search }\end{array}$ \\
\hline $\begin{array}{l}\text { Refinement is a social } \\
\text { process }\end{array}$ & $\begin{array}{l}\text { Document assemblers } \\
\text { Art, design \& architecture tools } \\
\text { User interface builders } \\
\text { Simulations, Models, } \\
\text { Templates, History, Macros }\end{array}$ \\
\hline $\begin{array}{l}\text { Creative work is not } \\
\text { complete until it is } \\
\text { disseminated }\end{array}$ & the field & $\begin{array}{l}\text { Listservs, Newsgroups } \\
\text { Conferencing, Groupware } \\
\text { Presentation, Annotation } \\
\text { Tele-democracy }\end{array}$ \\
\hline
\end{tabular}

Table 1: Genex framework: Foundational beliefs are tied to creative phases and sets of existing tools that can be refined and integrated into a genex.

\section{Genex: A generator of excellence}

The four foundational beliefs lead to a model of creativity with four phases and therefore four categories of tools. I hope this framework (Table 1) aids designers in building genexes that will enable creative individuals in many domains to:

- collect information from an existing domain of knowledge,

- create innovations using advanced tools,

- consult with peers or mentors in the field, and then

- disseminate the results widely.

The four phases of creativity-- collect, create, consult, and disseminate — can stimulate a research program to take current user interfaces to the next stage. The rapidly growing WorldWide Web is already a remarkable resource, but in the next fifty years it might grow by a factor of a thousand more users (from 107 to 1010) and a million times in data volume (from 108 pages to 1014 pages). These changes plus the increased expectations of users means that more effective designs will be needed.

Some innovations are incremental extensions of existing systems, but scaling up to cope with larger volumes of data/information/knowledge, increased numbers of users, and more ambitious endeavors will take some breakthroughs. For example, the design of an integrated family of tools requires some standardization of data interchange formats. The current situation is deteriorating as the proliferation of formats reduces the capacity of users to share their work. For a recent book project I received figures in 21 different formats, which required hours of effort by skilled professionals to unravel. Common industry standards, or at least self-describing data formats, will benefit users and producers, but it will take informed customer pressure and visionary technical leadership to find successful solutions. The successes of ASCII text, Unix files, Library of Congress MARC records, and TCP/IP network protocols are testimonies to the benefits of standardized data formats. The continued success of fax is an indictment of our failure to make standardized electronic mail commonplace. Sending images and structured data in email should be easier and more reliable than it is.

In addition to greater standardization of data, an integrated family of direct manipulation tools would have to support coordinations across multiple windows. Cut-copy-paste and drag-and-drop across applications are good first steps, but improved coordinations such as synchronized scrolling, hierarchical browsing, and multiple window open/close could help make genex users more effective. Imagine dragging the three-dimensional design of an automobile engine into a parts supply application, which would produce a complete parts list, manufacturing sequence, and cost estimate. Imagine dragging spreadsheet entries that represent a mailing list onto a mapping application, which would show the geographical distribution of entries (Roth et al., 1996).

I believe that advanced user interfaces can be built by innovative extensions of the principles of direct manipulation and the Object-Action Interface Model (Shneiderman, 1998a). 


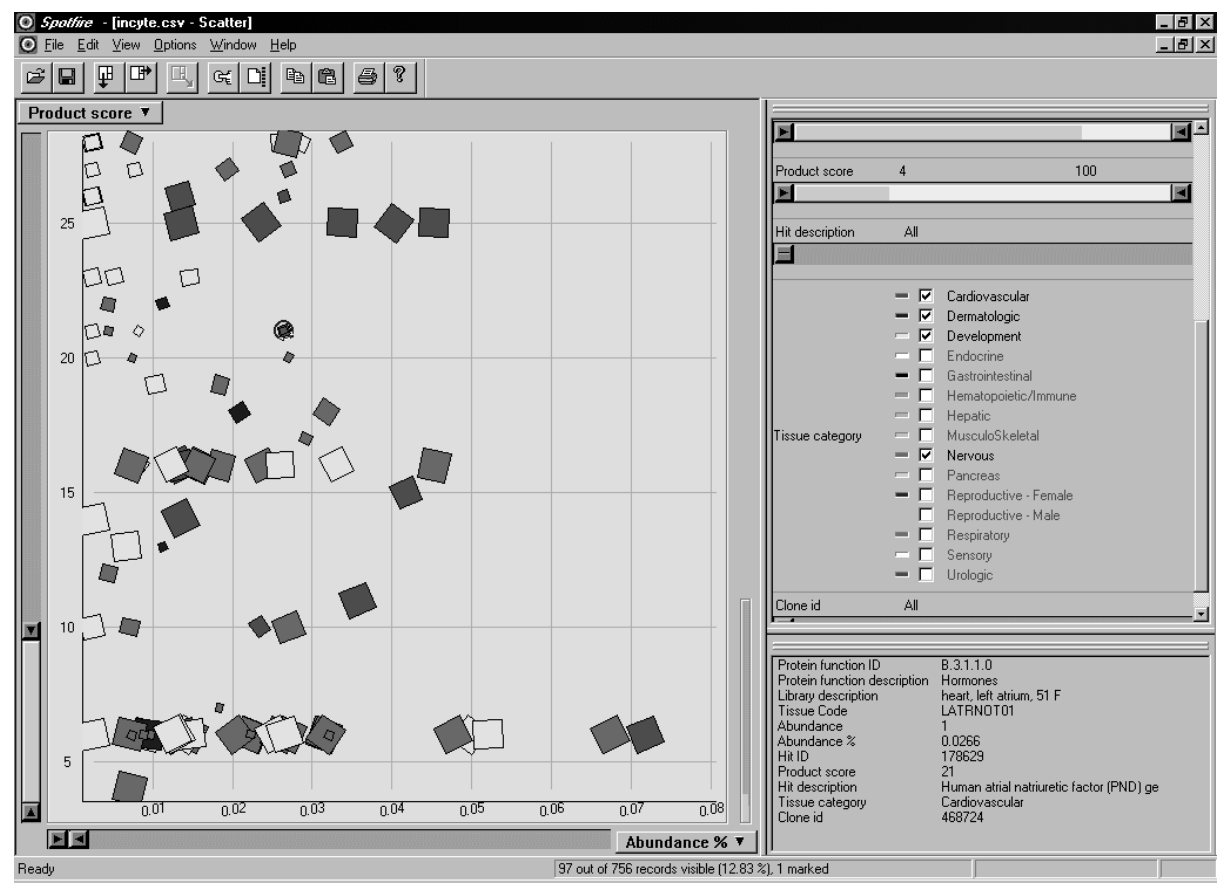

Figure 1: Spotfire visualization of human genome samples color coded by tissue type, where the user wants to identify strong contenders for doing biological testing in drug discovery. This starfield (color and size coded, zoomable scatterplot) display has user-controled dynamic query sliders (http://www.ivee.com).

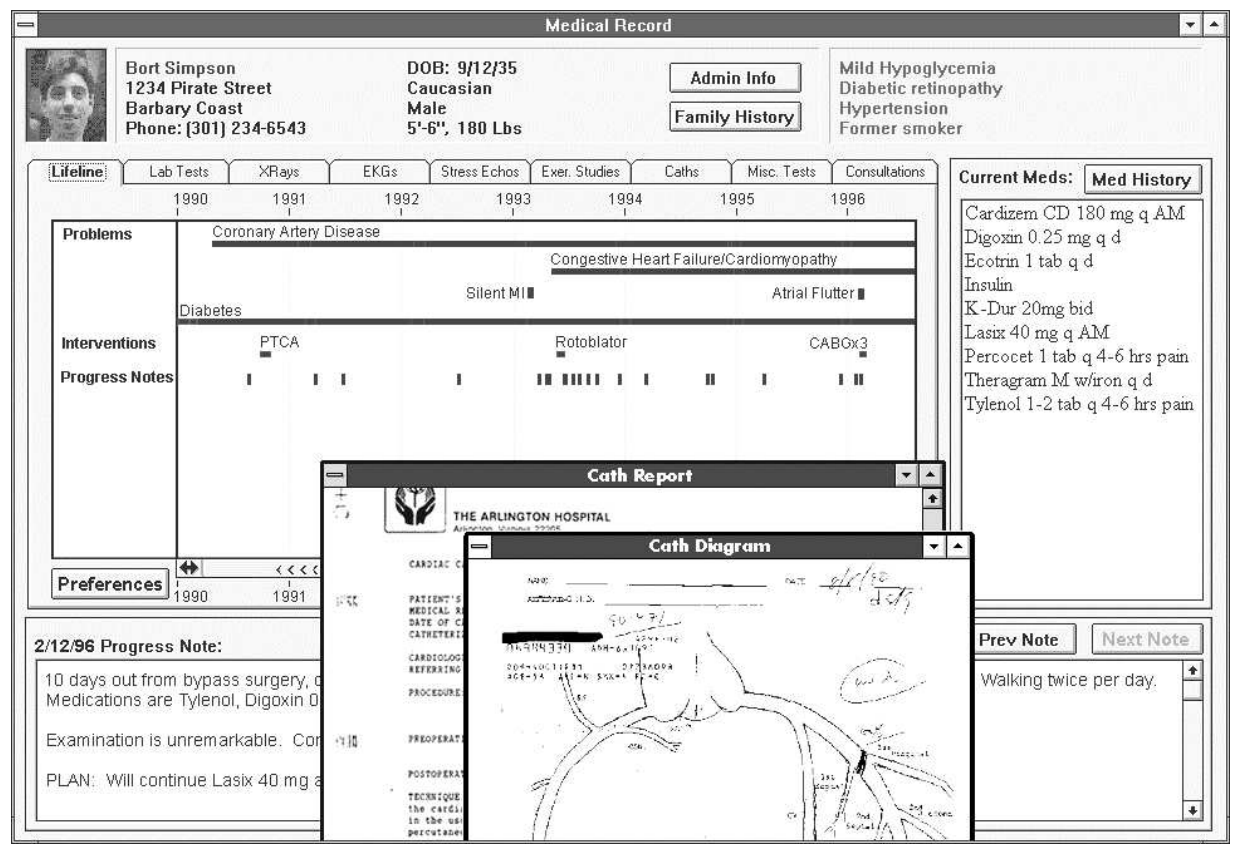

Figure 2: LifeLines is a visual presentation of a patient history with access to all supporting data (Plaisant et al., 1996). Website at http://www.cs.umd.edu/projects/hcil/Research/1997/patientrecord.html 
Users of higher resolution and larger displays will have access to large amounts of information, but they will need effective information visualization and manipulation tools to accomplish their increasingly ambitious goals (Robertson, Card and Mackinlay, 1993).

The challenges for designers are laid out in the following four sections. They connect foundational beliefs to a phase of creativity and then to a set of genex tools (Table 1).

\subsection{Collect information from an existing domain}

\section{of knowledge}

Finding relevant information and understanding it well enough to integrate it into existing knowledge requires intense commitment and concentration. The World Wide Web has made it possible to access large amounts of information, but finding, validating, and using it to construct useful knowledge are difficult tasks. The current attention to digital libraries is a positive step but increased emphasis on user interface design that supports the social construction of knowledge is still needed. Improved information design, search services, social structures, and learning skills can make dramatic differences (Marchionini, 1995).

Web pages are currently produced by low-level HTML statements that could be extended to contain more explicit semantics and structural information. Authors could indicate whether they are producing a product description with an animated demonstration, an annotated resume, a photo with a caption plus indexing information, or a scientific article. Richer document description and structuring languages will facilitate generation, sharing, and searching of complex medical histories, architectural drawings, or patents.

Search interfaces that allow more explicit statement of facets such as the scope of the search would enable users to focus on specific databases, topics, sources, languages, years, etc. (Shneiderman, Byrd and Croft, 1997). Person, geographic, and product name searches can all be improved by more explicit declaration of semantics in queries and web pages (Luke and Hendler, 1997). User control over phrase-based searches with stemming, spell correction, soundex matching, or partial word match can also support improved searching, as would increased control over the content, clustering, and ordering of search results. Visual presentations of thousands of results rather than a scrolling window with twenty items will improve the users' understanding of the search space, and their capacity to carry out query refinement steps.

Graphic user interfaces with dynamic queries for expressing searches and information visualization strategies for viewing results have the potential to improve search services (Ahlberg and Shneiderman, 1994; Shneiderman, 1994). A typical display might show publication date along the x-axis, and importance of documents along the y-axis. Size and color coding could show other variables such as length of documents or categories (Business, Sports, Arts, International). Alternatively, users could specify displays that show their interests, for example, relevance to Sports along the $\mathrm{x}$-axis and Business along the $\mathrm{y}$-axis. Stories about construction of a new sports stadium or sale of a baseball team would appear along the diagonal.

Searching in multimedia libraries is a further challenge. Some researchers believe that minimally-indexed collections can be searched by image, speech, or video content, but increased effort to capture searchable indexing information seems more potent. Collection of information is often a social process, and trusted authorities are needed to categorize and index knowledge for the whole web (for example, Yahoo) and in each disciplinary domain.

Social structures can also be helpful in facilitating collection of knowledge. Studies of information flow in organizations suggests that the primary sources are usually trusted colleagues who are known to be gatekeepers for knowledge in specific domains (Allen, 1977). Librarians have a vital role to play as indexers of information and then as knowledgeable facilitators during search. Another promising direction is the social process of asking for assistance in rapidly emerging online communities tied to specific domains. These are proving to be remarkably rapid and successful in helping some users, but scaling up to serve larger numbers of users reliably is a challenge (Ackerman and Palen, 1996).

Learning how to learn is often listed as a goal of education, but acquiring the goal-directed discipline, critical thinking skills, and cognitive self-awareness that support collection of knowledge is difficult. Advanced user interfaces may be able to help users better formulate their information needs, identify what information gaps impede them, and fabricate plans to satisfy their needs. Often as information is acquired, the user's knowledge shifts enough to require a reformulation of their plans. Information visualization interfaces and hypertext environments are a first step in supporting incidental learning, exploratory browsing, and then rapid reformulation of plans. As a refined theory of knowledge acquisition emerges, improved tools will follow.

\subsection{Create innovations using advanced tools}

Creativity is a demanding personal journey that follows diverse paths. General purpose tools such as word processors, spreadsheets, programming languages, and database systems are already helpful, and they can be extended. For example, word processors have grown to include spell checkers, grammar checkers, thesauri, outliners, indexers, etc. More sophisticated tools include style managers that allow easy conversion of the visual design and layout, and templates to facilitate construction of standard documents such as resumes, newsletters, or scientific papers. More elaborate document assemblers might apply complex rules to integrate 
components into tailored documents such as contracts, legislation, or corporate annual reports.

Future versions of general purpose tools can facilitate idea formation, brainstorming, argument or plot development, and comparison with existing ideas. Thesauri are to words, as genexes will be to ideas. Genexes will allow users to explore relationships among concepts, chemical molecules, or paintings by information visualizations (Ahlberg and Shneiderman, 1994) (Figure 1). These overviews would reveal clusters, gaps, outliers, and correlations, with easy linking to allow rapid exploration. Users could propose innovations or novel combinations (even random ones) and discover how they relate to existing ideas. For example, the components of a legal brief or movie script could be arranged on one of many standard scaffolds and then the genex could guide the user in making a complete document. Of course, users are still the source of the insight that makes a complete document also a compelling document.

Specialized tools can be even more effective in supporting creative work.. Every domain can have its electronic loom or lathe. For example, architecture analysis tools can verify structural integrity, estimate costs, and produce material ordering lists. Tomorrow's architects will be able to combine an appealing exterior design from one project with the interior space needs of the current client while computing structural engineering requirements. They will be able to make rapid changes to entire sections of an apartment complex, for example, doubling the sound insulation, air conditioning, or electrical capacity. Graphic artists will use computers to borrow existing or quickly construct reliable color palettes to achieve eye-catching effects or adjust sliders to increase/decrease symmetry, while working within their constraints of space and mood.

Simulations and models with three-dimensional or other animations are beginning to provide the flexibility that users need for creative explorations. Commercial successes such as the SimCity family demonstrate how rich scenarios can be made accessible to many users in a game-like style. Realistic simulations are far more complex and require substantial domain expertise, but they can be made accessible to motivated users. Direct manipulation programming should enable users to construct appropriate models even for complex situations and then macros enable automatic exploration of the state space. History keeping in simulations will enable users to review their work, share it with others, and compare alternate solutions. Design environments for manufacturing, engineering, and landscaping have begun to transform these domains, but the linkage to databases of existing designs and to computer supported cooperative work for consultation is still weak. Toolkits for composing music and art can allow users to begin by taking existing works or generic templates. Novices will have the capacity to make constrained exploratory variations, while experts will have an enormous range of capabilities for complex manipulations. Decision support tools start with checklists and standard situations, but can become elaborate environments in which users discover interesting relationships, annotate anomalies, and add meta-rules for future users.

Improved designs will shorten learning times and provide guides to novice users. An example to build on is Adobe PhotoDeluxe, which leads users through the multiple steps necessary for editing and retouching photos. Future tools will provide standardized learning streams to help novices perform basic tasks and scaffolding that wraps the tool with guidance as users acquire expertise. Experts will be able to record their insights for others and make macros to speed common tasks by novices.

A major advance in user interfaces that supports creative exploration would the capacity to go back in time, to review and manipulate the history of actions taken during an entire session. Users will be able to see all the steps in designing an engine and change an early design decision. They will be able to extract sections of the history to replay them or to convert into permanent macros that can be applied in similar situations. Extracting and replaying sections of history is a form of direct manipulation programming. It enables users to explore every alternative in a decision-making situation, and then chose the one with the most favorable outcomes. Remember, those who ignore history, are destined to retype it.

\subsection{Consult with peers or mentors in the field}

Consultation by electronic environments is already speeding up and raising quality in many domains. Rapid progress in the web development community is partially a product of the ease with which people can post their current efforts for others to examine, test, and comment. Feedback is rapid and constructive, but sometimes brutal. Security holes produce international attention and lead to new versions in days or sometimes hours. Reviewers' comments on new software appear rapidly and evince high expectations of swift progress. The danger of working at "internet time" is that hasty decisions may be poor, and rapid changes may cause troubling turbulence for many users.

In other fields the process of consultation is formalized such as in the review process for scientific papers. Submissions to journal editors are distributed, often anonymously, to appropriate reviewers for comments and suggested revisions. Acceptable manuscripts are usually reviewed a second and possibly a third time before entering the production process that leads to a published journal article. The entire process can take 12-36 months. Clearly, there is room for speed-up and improvement of the review process if electronic submissions and annotated reviews can be arranged. This is still rare because the technology does not conveniently support document construction and sharing, when figures, photos and formulas are required. Genexes would support prompt 
reviews with tools for journal editors to track status and chase down recalcitrant reviewers.

Other consultation technologies include basic tools such as email, listservs, newsgroups, chat rooms, and bulletin boards, and more elaborate MUDs and MOOs. These have been successful, but research is needed to understand the role of moderators, how search tools could be improved, and how these environments will scale up to a thousand times as many users (Kraut et al., 1994; Olson and Olson, 1997; Kiesler, 1997). A key issue is the role of empathic communications in forming trusting relationships (Preece, 1998). Audio/video conferencing is slowly gaining popularity, as early designs such as CUSeeMe mature (Dorcey, 1995) and comprehensive tools such as Microsoft NetMeeting emerge. Reliable network infrastructures that provide adequate bandwidth at low cost are increasingly available.

Telemedicine consultations could improve care in remote areas, and offer all patients the opportunity to have multiple reviewers of their lab tests or medical images. Specialists would have the advantage of seeing more cases that they are interested in, thereby facilitating experimental testing of new procedures or medications.

Distance education by tele-mentoring, tele-lecturing, and computer mediated conferencing is gradually reshaping education, and is likely to accelerate as the technology becomes more widely available. Additional research and development is needed to explore how education can be reshaped in a 24-hour electronic environment in which the teacher shifts from being the "sage on the stage to the guide on the side." The web supports collaborative teaching methods in which students do more than surf the net -- they learn to make waves. Ambitious team projects can provide valuable services to clients who are outside the classroom. These authentic projects can be highly motivating to students as they learn business-oriented and personally enriching skills of communicating, critiquing, and collaborating (Shneiderman, 1998b).

Important tools are still needed for group formation and discussion within communities of tens, thousands, and millions of people. Participation in democratic political processes are appealing, but ensuring informed participation, respect for opposing views, and adequate time for deliberation will be difficult. A major research effort would help to grapple with complex issues of thousand of active participants in discussion groups. How would an electronic Robert's Rules of meetings help to keep order, permit caucusing of subgroups, support voting, and allow objections to be aired?

\subsection{Disseminate the results widely}

An innovation is complete when it is disseminated to appropriate members of the field. A genex would enable innovators to reach large numbers of interested people rapidly and conveniently. Posting a new algorithm, poem, or video on the web makes it available, but unless appropriate recipients notice it, the originator has little chance to influence them.

Improved search engines will help for devoted searchers, but promoting a new contribution in the marketplace of ideas still takes effort. Improved methods to reach the right audiences will begin with email and newsgroups, but should be extended by novel group formation methods.

For example, as an author of scientific papers, I want to send my new work to all the people whom I reference. I also want to reach others interested in this topic so I might send my work to those who might declare an interest in a sub-domain or authors who have referenced the papers I reference. Another approach is to create a discussion group for every published paper. Readers of each paper would be invited to join the discussion group.

Creators of artistic works such as songs, poems, or paintings could also reach their audiences with improved online performances, anthologies, and galleries, respectively. These forms of electronic publications or narrowcasting would be supervised, moderated, or curated by knowledgeable critics who would promote quality and develop audiences or markets.

Email currently helps some people build affiliation networks of people with shared interests. Commercial email services are forming niche lists of selected subscribers to support electronic commerce, and similar services will help in the dissemination of creative work. Listervs, Newsgroups, and Bulletin Board Systems function adequately for groups of thousands of users, and refinement to reach millions is possible with advanced concepts of moderation, sub-group formation, and clustering of topics. Eventually Ecommunities of shared interest will germinate and grow for almost every topic, sprouting myriad diverse branches or gathering branches into new alliances.

A key barrier to dissemination of creative work is the desire to protect intellectual property and profit from copyright royalties and patent licensing. Intellectual property laws were always designed to promote dissemination of novelty, but owners have often acted to restrain utilization by excessive charges or by legal intervention. A combination of new technologies, social structures, and legal formulations might promote dissemination of ideas by ensuring fair royalties for all contributors. A happy outcome would be frequent creative chain reactions, in which one innovation triggers several others and quickly leads to the emergence of a new domain, breakthrough scientific discoveries, innovative engineering, and useful products. The electronic record of contributions would help ensure appropriate royalties and honor. 


\section{Genex Scenarios}

The four phases and sets of tools in the genex framework (Table 1) are meant to guide designers of advanced user interfaces. Genexes will be built for many domains, but a few examples may help to clarify how they will be used.

\subsection{Medical-diagnosis genex: Patient history libraries}

A medical genex could support the diagnostic process by providing access to the patient history and clinical databases of treatment outcomes, decision-making support, consultation with specialists, and logging of outcomes for future study. Compact presentation of relevant aspects of a patient's history, current signs and symptoms, laboratory results, medical images, etc. are the starting point for a diagnostic decision (Plaisant et al., 1996) (Figure 2).

When physicians are familiar with the case, they begin to create hypotheses and form potential treatment plans, but they can return to collecting more background information from a vast database of patient records. They can select samples of patients with similar characteristics (gender, age, weight, health, etc.) who have similar symptoms, and then study how outcomes are correlated with treatments. They might discover that surgery was more successful for younger patients, but chemotherapy is more successful for older patients. The physicians can then move on to creating a treatment plan using medical models that predict outcomes and check for contraindications or interactions with other ongoing treatments for this patient.

The physician can then consult by email or conferencing with the patient or experts in the field to gain support for the plan. As the treatment unfolds, lab results verify progress, and all the information is entered in the patient database for other physicians to consult in future cases. A medical diagnosis genex would also be the basis for clinical research to determine the efficacy of treatments and understand a wide variety of health issues: aging processes, spread of infectious diseases, environmental impact on health, etc. A controversial application would be the use of treatment information for evaluating physicians or hospitals.

Of course, personal privacy is vital for medical records and new policies will have to be developed. New techniques will have to be developed to ensure appropriate access and log usage. Even when every effort has been made to protect privacy, some breakdowns will occur, so legal remedies and compensation must be discussed as part of the system design.

\subsection{Educational project genex: On-web science festivals}

Contemporary science fairs encourage students to get deeply involved in one topic and produce a report, device, or experiment. They are motivated to impress a jury of teachers who review the projects and award prizes. This paradigm has value, but critics point out that guidance to students is often shallow, jurors have limited time to review, winning takes on more significance than learning, and the projects are seen by only a few people for a short period of time.

Imagine an on-web science festival that used a genex. Web pages could offer students templates for a variety of science projects, background information tailored to their age and knowledge with links to related web sites, and online communities of peers and mentors working in their area of interest (Shneiderman, 1998b). For creative students, a template-breaking process would also be included.

Once they chose a project direction, learner-centered inquiry tools might support hypothesis formulation, simulation or real-world experiments, mathematical modeling, and scientific data collection and analysis (Soloway et al., 1996). More specific domain-related tools might enable environmental pollution tracking, genetic data, or sociological survey administration.

As they progress in their work, comments from peers and mentors can help shape their initial hypotheses, proposed experiments, and construction diagrams while improving their on-web presentations. Feedback about the work and its presentation will lead to further polishing until it is ready to be presented to wider circles of students, teachers, mentors, and jurors. Jurors, in concert with teachers and mentors, may also designate a fraction of the projects for preservation on the web so that they can become a resource for future science festival students. The dissemination of projects means that over time student projects should build on each other and gain in sophistication. Publishing projects on the web may increase motivation because so many others may see their work. This was my experience in requiring semester-long team projects on empirical user-interface studies to be published on the web in my senior undergraduate course http://otal.umd.edu/ SHORE). Another example is the valuable scientific resource on information visualization produced during a graduate seminar (http://otal.umd.edu/Olive).

\subsection{Facilities-design genex: Electronic classroom guidetool}

I was recently worked with a contractor who was asked to write a guidebook for designers of electronic classrooms that would contain detailed information on floorplans, computer desks, networks, projectors, lighting, air-conditioning, etc. and related educational strategies. This codex-like outcome was upgraded to a memex-like outcome by changing the requirement that the guidebook be available on the web.

Applying the genex framework led to a more ambitious goal; building an interactive environment that enables facilities designers to create architectural drawings, lists of equipment, and a schedule for ordering and installation. This guidebook could become a guidetool (documents become user interfaces) that allows facilities designers to explore alternate layouts to see how many desks, computers, and chairs could fit into the available space and budget. Two-dimensional drawings and 
three-dimensional VRML virtual environments could be examined and experienced to give a clear understanding of what the classroom will look like.

When a design is chosen, it can then be made available electronically to other decision-makers, potential builders, and equipment suppliers for their comments and bids. Once the room is built, the plans could be archived for future designers to review and use as a starting point for their even more advanced designs.

\subsection{Legislation-writing genex: THOMAS 2.0}

The U. S. Library of Congress built the THOMAS (a tribute to Thomas Jefferson) web site to provide public access to legislative information, including the full text of bills before the U. S. Congress. It has been a great success with ten million visits per month by journalists, lobbyists, scholars, and students interested in specific legislation. Popular topics change weekly, but abortion bills, handgun control, welfare reform and other contemporary issues have been near the top of the list for users of this memex-like tool.

Upgrading from memex to genex is a natural next step. An upgrade to THOMAS 2.0 might include tools for informed citizens' groups and lobbying organizations to better understand the legislative process and participate appropriately. They might explore the need for new legislation, contact staffers of interested members of Congress, compare previous legislation by contents, and contact relevant professional societies to gain support. For activist citizen groups and legislative counsels who draft bills, there could be templates that would guide the process and ensure proper terminology, appropriate phrasing, and adherence to legal requirements. Then when first drafts were completed, the consultation process would begin with supporters and potential opponents. As revisions are made, key partners would be informed of the latest changes, and a predictive model of how many votes were still needed could guide further revisions. As committee meetings, hearings, and the final markup progresses, key players would be kept informed of changes and potential concerns.

Democratic processes take time. The goal of a legislationwriting genex is not necessarily to speed the process or increase the number of bills, but to engage a wider circle of stakeholders, support thoughtful deliberation, and improve the quality of the resulting legislation.

\section{Conclusion and Cautions}

Creativity is often described as a rare experience for elite workers, but genexes can promote contributions from more diverse individuals to ever widening domains of knowledge. Initial steps are appearing on the World Wide Web, there is little coordination and many designs lack vital components. This genex framework is meant to encourage development of an integrated family of tools for creative work by enabling individuals to:

- collect information from an existing domain of knowledge,

- create innovations using advanced tools,

- consult with peers or mentors in the field, and then

- disseminate the results widely.

Many versions of genex are likely to be built, capturing the history of each domain, tailored to the needs of each field, and in harmony with the working styles of diverse individuals. Some genexes are likely to be too restrictive in their support of creativity or too limited in their functionality, but the examples of excellence should help to promote quality. Progress in implementing a genex will be measured by the number of adopters and their productivity, but progress as a civilization will be harder to measure.

No one can guarantee that genex technologies will be applied in positive ways, but novel social structures that encourage participatory design and open discussions may more regularly lead to higher societal benefits. Each professional's contribution to societal transformation becomes an inspiration and he or she becomes a role model for others. Every story we tell about one person making a difference inspires the next constructive contribution.

Thoughtful commentaries about how to design (Friedman, 1997), implement, and manage technology are beginning to appear in the form of ethical codes of conduct from professional societies such as the ACM and the IEEE (Gotterbarn, Miller and Rogerson, 1997). These are intended to reduce negative side effects by encouraging professionals to:

"...minimize negative consequences of computing systems, including threats to health and safety...avoid harm to others...take action not to discriminate...respect the privacy of others." (http://www.acm.org/constitution/ bylaw17.html)

We live in a troubled world, with immense problems that are not caused by technology: conflict and oppression, poor health and nutrition, inadequate housing or sewage, illiteracy and poor education, and many more. Computing and communications technology may contribute to solutions by enabling people to explore a wider set of alternatives, consult easily with peers and mentors, and record their successes (and failures) so others can learn from them.

Our greatest resource is human creativity. Appropriate technology can empower more people to experience the thrill of creative work and enable each of us to make a difference.

\section{Acknowledgments}

Thanks to Gavriel Salvendy and Michael Smith for their support, encouragement, and commentary. I greatly 
appreciated the invitation to keynote the Human-Computer Interaction '97 Conference and to present this paper in the International Journal of Human-Computer Interaction. Thanks also for comments from Maryle Ashley, Amy Friedlander, James Hendler, Svetlana Knizhnik, Jaron Lanier, Gary Marchionini, Kent Norman, Christopher North, Jenny Preece, and Mitchell Waldrop.

\section{References}

Ackerman, Mark S. and Palen, Leysia, The Zephyr help instance: Promoting ongoing activity in a CSCW system, Proc. CHI'96 Human Factors in Computer Systems, ACM, New York, NY (1996), 268-275.

Ahlberg, Christopher and Shneiderman, Ben, Visual information seeking: Tight coupling of dynamic query filters with starfield displays, Proc. CHI94 Conference: Human Factors in Computing Systems, ACM, New York, NY (1994), 313-321 + color plates.

Allen, Thomas J., Managing the Flow of Technology, MIT Press, Cambridge, MA (1977).

Berners-Lee, Tim, Cailliau, Robert, Luotonen, Ari, and Nielsen, Henrik, F., and Secret, Arthur The world wide web, Communications of the ACM 37, 8 (1994), 76-82.

Brooks, Frederick, Jr., The computer scientist as toolsmith II, Communications of the ACM 39, 3 (March 1996), 61-68.

Bush, Vannevar, As we may think, Atlantic Monthly 76, 1 (July 1945), 101-108. Also at http://www2.theAtlantic.com/ atlantic/atlweb/flashbks/computer/tech.htm

Dorcey, Tim, CU-SeeMe desktop videoconferencing software, Connexions 9, 3 (March 1995). Also at http://cuseeme.cornell.edu/DorceyConnexions.html.

Engelbart, Douglas C. and English, William K., A research center for augmenting human intellect, AFIPS Proc. Fall Joint Computer Conference 33 (1968), 395-410.

Friedman, Batya (Editor), Human Values and the Design of Computer Technology, CSLI Publications, Stanford, CA and Cambridge Univ. Press, UK (1997).

Gotterbarn, Don, Miller, Keith, and Rogerson, Simon, Software engineering code of ethics, Communications of the ACM 40, 11 (1997), 110-118.

Kiesler, Sara (Editor), Culture of the Internet, Lawrence Erlbaum Assoc., Inc., Mahwah, NJ (1997).

Kraut, Robert E., Cool, Colleen, Rice, Ronald E., and Fish, Robert S., Life and death of new technology: Task, utility and social influences on the use of a communications medium,
Proc. Conference on Computer Supported Cooperative Work '94, ACM, New York, NY (1994), 13-21.

Licklider, J. C. R., Libraries of the Future, MIT Press, Cambridge, MA (1965).

Luke, Sean and Hendler, James, Web agents that work, IEEE Multimedia 4, 3 (July-Sept 1997), 76-79.

Marchionini, Gary, Information Seeking in Electronic Environments, Cambridge University Press, UK (1995).

National Research Council - Committee on a National Collaboratory, National collaboratories: Applying information technology for scientific research, National Academy Press, Washington, DC (1993).

Olson, Gary M. and Olson, Judith S., Research on computer cooperative work, In Helander, M. G., Landauer, T. K., and Prabhu, P.V. (Editors), Handbook of Human-Computer Interaction: Second Edition, Elsevier, Amsterdam (1997), 1433-1456.

Plaisant, Catherine, Rose, Anne, Milash, Brett, Widoff, Seth, and Shneiderman, Ben, LifeLines: Visualizing personal histories, Proc. of ACM CHI'96 Conference: Human Factors in Computing Systems, ACM, New York, NY (1996), 221227,518 .

Preece, Jenny, Empathic communities: Balancing emotional and factual communications, Interacting with Computers (1998, to appear).

Robertson, George G., Card, Stuart K., and Mackinlay, Jock D Information visualization using 3-D interactive animation, Communications of the ACM 36, 4 (April 1993), 56-71.

Roth, Steven F., Lucas, Peter, Senn, Jeffrey A., Gomberg, Cristina C., Burks, Michael B., Stroffolino, Philip J., Kolojejchick, John A. and Dunmire, Carolyn, Visage: A user interface environment for exploring

information, Proc. Information Visualization '96, IEEE, Los Alamitos, CA (1996), 3-12.

Shneiderman, Ben, Dynamic queries for visual information seeking, IEEE Software 11, 6 (1994), 70-77.

Shneiderman, Ben, A grander goal: A thousand-fold increase in human capabilities, Educom Review 32, 6 (November 1997), 4-10.

Shneiderman, Ben, Designing the User Interface: Strategies for Effective Human-Computer Interaction: Third Edition, Addison-Wesley Publ. Co., Reading, MA (1998a) 
Shneiderman, Ben, Relate-Create-Donate: A teaching philosophy for the cyber-generation, Computers \& Education (1998b, to appear).

Shneiderman, B., Byrd, D., and Croft, B., Clarifying search: A user-interface framework for text searches, D-Lib Magazine http://www.dlib.org/dlib/january97/01 contents.html (January 1997).

Shneiderman, Ben and Kearsley, Greg, Hypertext HandsOn! An Introduction to a New Way of Organizing and Accessing Information, Addison-Wesley, Reading, MA (1989).

Soloway, Elliot, Jackson, Shari L., Klein, Jonathan, Quintana, Chris, Reed, James, Spitulnik, Jeff, Stratford, Steven J., Studer, Scott, Jul, Susanne, Eng, Jim and Scala, Nancy, Learning theory in practice: Case studies of learnercentered design, Proc. ACM CHI'96 Conference, ACM, New York, (1996), 189-196. 